\title{
Some physiological characteristics of neomycin and kanamycin-resistant strains of Staphylococcus aureus
}

\author{
S. I. JACOBS ${ }^{1}$ AND A. T. WILLIS ${ }^{2}$ \\ From the Department of Bacteriology, School of Medicine, Leeds
}

SYNOPSIS Five hundred and fifty-two strains of Staphylococcus aureus of hospital origin were resistant to penicillin, streptomycin, and tetracycline. Of these, 298 were also resistant to neomycin. and kanamycin, and this resistance was related to pigment production on glycerol monoacetate $\sigma_{-}$ agar, the production of $\beta$-lysin, the absence of fibrinolytic and proteolytic activity, and to phage $\vec{N}$ susceptibility. The use of physiological markers, the inadequacy of phage typing, and the possible을 reasons for the emergence of neomycin-resistant staphylococci are discussed.

During 1963 the appearance in hospitals of new epidemic varieties of Staphylococcus aureus was noted by Temple and Blackburn (1963), Jacobs, Willis, Ludlam, and Goodburn (1963), Willis, Jacobs, and Goodburn (1963), Turner (1963), and Robertson (1963). These organisms, which are difficult to type with the standard set of staphylococcal phages, are characterized by the yellow pigmentation of their colonies on glycerol monoacetate agar (Willis and Turner, 1962), and by their resistance to penicillin, streptomycin, and tetracycline. The strains described by Temple and Blackburn showed inhibition reactions at 1,000 R.T.D. with phages $7 / 47 / 53 / 54 / 75 / 77$, and they referred to these organisms as phage 'type $A$ '. Robertson used the same nomenclature, but Jacobs et al. drew attention to the fact that inhibition reactions are not sufficiently reproducible to form the basis of a pattern for the recognition of a staphylococcal phage type. They were of the opinion that these organisms should be designated 'non-typable'. Although the exact phage status of these staphylococci has not yet been determined. it seems that they form part of a group of related organisms linked to those of phage group III, which have other characteristics in common. They almost always form yellow pigmented colonies on glycerol monoacetate agar, they are resistant to penicillin, streptomycin, and tetracycline, and are non-proteolytic. In addition, a high

'Department of Pathology, Booth Hall Children's Hospital, Manchester 9.

'Department of Microbiology, Monash University Medical School, Alfred Hospital, Melbourne, Australia.

Keceived for publication 21 January 1964. proportion are non-fibrinolytic, and many strains $\frac{\mathbb{1}}{3}$ produce $\beta$-lysin (Willis et al., 1963, 1964).

Strains similar to these have a world-wide distri- $\overrightarrow{0}$

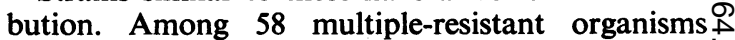
kindly sent to us by Dr. Jean Tolhurst and Mr. Glen Buckle from Melbourne, Australia, we found that 16 were of the yellow multiple-resistant, nonproteolytic variety. Likewise, Goodburn (1963) found that organisms of this type form a high pro- $\mathbb{D}$ portion of the multiple-resistant staphylococci which she has isolated in Perth, Western Australia. Cohen, Fekety, and Cluff (1962) described epidemic strains of Staph. aureus at the Johns Hopkins Hospital, Baltimore, which had phage and sensitivity characteristics somewhat similar to the British isolates, and it can be predicted with reasonable certainty that theo American organisms will possess the other physiological features common to the British group. Cohen and his co-workers were impressed by the frequency with which their strains were resistant to kanamycino and neomycin, and they attributed this to the greatly increased use of neomycin in the pre-operative? preparation of the bowel at their hospital. Robertson has shown that the type $A$ strains of Temple and $O$ Blackburn are also resistant to kanamycin and N neomycin, but Jacobs and Willis (1963) extended N these observations to the yellow group as a whole. 0 They showed that resistance to these antibiotics is a general and almost exclusive property of yellow, tetracycline-resistant staphylococci, and is not: limited to the type $A$ strains of Temple and Blackburn.

The present study is concerned with 552 strains of tetracycline-resistant staphyloccoci, and draws atten- $\overrightarrow{\mathrm{D}}$ tion to the relationship that exists between resistance $\frac{2}{2}$ 612 
to kanamycin and neomycin on the one hand, and pigmentation, phage susceptibility, $\beta$-lysin production, fibrinolytic activity, and proteolysis on the other.

\section{EXPERIMENTAL}

STRAINS OF STAPHYLOCOCCI The 552 strains of Staph. aureus were recently cultured from the in-patients of two large general hospitals. They were isolated from septic lesions over a period of six months and they represent that group of organisms that was resistant to penicillin, streptomycin, and tetracycline within a much larger series of consecutively isolated staphylococci. The remainder were all sensitive to tetracycline, virtually all to streptomycin, and many were sensitive to penicillin.

In addition to the $\mathbf{5 5 2}$ tetracycline-resistant organisms with which this investigation is mainly concerned, 50 tetracycline-sensitive strains of Staph. aureus were also studied; 25 of these were sensitive to penicillin and the remainder were resistant.

SENSITIVITY TESTS These were performed by the disc diffusion method using Multodisks (Oxoid) which contained penicillin 1.5 units, streptomycin, chloramphenicol, tetracycline and erythromycin, $10 \mu \mathrm{g}$., and novobiocin $5 \mu \mathrm{g}$. In addition, all strains were tested for sensitivity to methicillin (Celbenin) with $10 \mu \mathrm{g}$. discs (Mast Laboratories), cloxacillin (Orbenin) using $5 \mu \mathrm{g}$. discs (Beecham Research Laboratories), and fusidic acid (Fucidin) with $10 \mu \mathrm{g}$. discs (Leo Laboratories). Sensitivity to neomycin was determined with $10 \mu \mathrm{g}$. discs (Mast Laboratories), and to kanamycin with discs containing $7.5 \mu \mathrm{g}$. of the antibiotic prepared by the method described by Willis and Turner (1963).

Cultural Characteristics Pigmentation was determined from 48-hour cultures on glycerol monoacetate agar (Willis and Turner, 1962). (For details of the preparation of this medium, see Jacobs, Willis, and Goodburn, 1964.) Fibrinolytic activity and proteolysis were determined on human thrombin-fibrinogen agar and on the washed chocolate agar medium described by Jacobs et al. (1964). Production of $\beta$-lysin was determined using cultures on an agar medium containing $10 \%$ washed sheep erythrocytes (Burroughs Wellcome and Co.). These were incubated under anaerobic conditions for $\mathbf{4 8}$ hours at $37^{\circ} \mathrm{C}$., and then cooled to $4^{\circ} \mathrm{C}$. for one hour before reading.

\section{RESULTS}

All strains that were resistant to neomycin were also resistant to kanamycin; similarly, strains that were neomycin-sensitive were also inhibited by kanamycin. In the following data, therefore, we have found it convenient to express the results in terms of one antibiotic only, namely, neomycin.

None of the 50 tetracycline-sensitive strains of Staph. aureus was resistant to neomycin. On the other hand, 298 of the 552 tetracycline-resistant strains were also resistant to neomycin.
Table I shows that 378 of the 552 tetracyclineresistant organisms were of the yellow variety, and the remaining 174 were either orange or buff. Of the 378 yellow pigmented organisms, $295(78.0 \%)$ were resistant to neomycin. On the other hand, only three of the 174 orange and buff strains $(1.7 \%)$ were neomycin-resistant. Thus, 295 out of 298 neomycinresistant strains $(95.6 \%)$ were of the yellow variety.

\section{TABLE I}

RELATIONSHIP BETWEEN PIGMENTATION, NEOMYCIN RESISTANCE, AND PHAGE

SUSCEPTIBILITY OF 552 TETRACYCLINE-RESISTANT STRAINS OF STAPHYLOCOCCUS AUREUS

Pigment Group

\begin{tabular}{llcr}
\hline Colour & $\begin{array}{l}\text { 'Phage } \\
\text { Susceptibility }\end{array}$ & $\begin{array}{c}\text { Neomycin- } \\
\text { sensitive }\end{array}$ & $\begin{array}{c}\text { Neomycin- } \\
\text { resistant }\end{array}$ \\
\hline Yellow & R.T.D. & 22 & 25 \\
& 1,000 R.T.D. & 55 & 124 \\
Orange and buff & Not typable & 6 & 146 \\
& R.T.D. & 102 & 0 \\
& 1,000 R.T.D. & 48 & 2 \\
& Not typable & 21 & 1
\end{tabular}

It is clear, therefore, that neomycin resistance is shown mainly by staphylococci that produce yellow pigment on glycerol monoacetate agar. The results summarized in Table I also show that resistance to neomycin amongst these yellow organisms is not limited to non-phage-typable strains, i.e., the staphylococci designated type A by Temple and Blackburn and later by Robertson, but is also possessed by strains that are typable both at R.T.D. and at 1,000 R.T.D. Although resistance to neomycin is displayed almost exclusively by yellow staphylococci, by no means all of them are resistant. Thus, in our series, $83(21.9 \%)$ were sensitive. However, it is of some interest to note that amongst the nontypable strains, only six out of $152(3.9 \%)$ were neomycin-sensitive, but amongst the organisms typable at R.T.D. 22 out of $47(46 \cdot 8 \%)$ were inhibited by this antibiotic. Those strains typable only at 1,000 R.T.D. occupied a position intermediate between these, about $31 \%$ of them being sensitive.

Table II shows the relationship between neomycin resistance and proteolysis on washed chocolate agar. Willis et al. $(1963,1964)$ have shown that only a few yellow tetracycline-resistant staphylococci are proteolytic on this medium, in contrast to orange and buff organisms, amongst which some $60 \%$ are actively proteolytic. Because neomycin resistance is largely limited to yellow tetracylineresistant organisms, it follows that neomycin-resistant strains are usually non-proteolytic. However, inability of a strain to cause clearing on the washed chocolate agar does not provide an index either of its pigment variety or its neomycin resistance. Thus, 
TABLE II

RELATIONSHIP BETWEEN PIGMENTATION, NEOMYCIN RESISTANCE, PHAGE SUSCEPTIBILITY, AND PROTEOLYSIS OF 552 TETRACYCLINE-RESISTANT STRAINS OF STAPHYLOCOCCUS AUREUS

\begin{tabular}{|c|c|c|c|c|c|}
\hline \multicolumn{2}{|l|}{ Pigment Group } & \multicolumn{2}{|c|}{ Neomycin-sensitive } & \multicolumn{2}{|c|}{ Neomycin-resistant } \\
\hline Colour & Phage Susceptibility & Proteolytic & Non-proteolytic & Proteolytic & Non-proteolytic \\
\hline \multirow[t]{3}{*}{ Yellow } & R.T.D. & 0 & 22 & 0 & 25 \\
\hline & 1,000 R.T.D. & 0 & 55 & 0 & 124 \\
\hline & Not typable & 0 & 6 & 0 & 146 \\
\hline \multirow{3}{*}{ Orange and buff } & R.T.D. & 87 & 15 & 0 & 0 \\
\hline & 1,000 R.T.D. & 8 & 40 & 0 & 2 \\
\hline & Not typable & 2 & 19 & 0 & 1 \\
\hline
\end{tabular}

TABLE III

RELATIONSHIP BETWEEN PIGMENTATION, NEOMYCIN RESISTANCE, PHAGE SUSCEPTIBILITY, FIBRINOLYSIS, AND $\beta$-LYSIN PRODUCTION OF 522 TETRACYCLINE-RESISTANT STRAINS OF STAPHYLOCOCCUS AUREUS

Pigment Group Neomycin-sensitive Neomycin-resistant

\begin{tabular}{|c|c|c|c|c|c|}
\hline Colour & Phage Susceptibility & Fibrinolytic & Non-fibrinolytic & Fibrinolytic & Non-fibrinolytic \\
\hline \multirow[t]{4}{*}{ Yellow } & R.T.D. & 20 & 2 & 3 & $\begin{array}{c}22 \\
(21 \beta+v e)\end{array}$ \\
\hline & 1,000 R.T.D. & 48 & 7 & 15 & $\begin{array}{c}109 \\
(93 \beta+\mathrm{ve})\end{array}$ \\
\hline & Not typable & 5 & 1 & $\left(3 \beta^{72}+\mathrm{ve}\right)$ & $\begin{array}{c}74 \\
(14 \beta+\text { ve })\end{array}$ \\
\hline & R.T.D. & 88 & 14 & 0 & 0 \\
\hline \multirow{2}{*}{ Orange and buff } & 1,000 R.T.D. & 45 & 3 & 2 & 0 \\
\hline & Not typable & 18 & 3 & 1 & 0 \\
\hline
\end{tabular}

out of 455 non-proteolytic strains, $157(34 \cdot 5 \%)$ were sensitive to neomycin, and of these $74(47 \cdot 1 \%)$ were of the orange or buff variety. Amongst the orange and buff strains there is an interesting relationship between proteolysis on the one hand and phage susceptibility on the other. Strains typable at R.T.D. showed a ratio of proteolysis to non-proteolysis of 6 to 1 , but the same ratio calculated for strains typable at 1,000 R.T.D. was 1 to 5, and that of nontypable strains was 1 to 10 . Thus, pari passu with increasing resistance to phage lysis there is diminishing proteolytic activity, both of which features are considered by Jacobs et al. $(1963,1964)$ to be characteristic of saprophytic staphylococci.

Table III shows the relationship of neomycin resistance to fibrinolytic activity and production of $\beta$-lysin. It emphasizes the correlation between resistance to neomycin and inability to attack fibrin. Thus, among the yellow neomycin-resistant organisms, 205 of $295(69.4 \%)$ were non-fibrinolytic, but in the neomycin-sensitive group only 10 of $83(12 \%)$ failed to attack this substrate. Again, only $12 \%$ of the orange and buff neomycin-sensitive strains were non-fibrinolytic. In view of these findings, it can be said with some certainty that if an organism is found to be yellow on glycerol monoacetate agar and is non-fibrinolytic, then it will also be resistant to neomycin. This association is even greater when the non-typable, neomycin-resistant strains are excluded from consideration. Then, of 149 phage- susceptible neomycin-resistant strains, $131(87.9 \%$ were non-fibrinolytic. It is clear, therefore, tha amongst phage-susceptible strains, absence oहै fibrinolytic activity is linked with neomycin resis $\underset{\mathbb{Q}}{\square}$ tance. This suggested separation of the non-typable yellow neomycin-resistant strains into a differeng catagory is not unreasonable, because the ratio of fibrinolytic to non-fibrinolytic organisms in thiș. group is 1 to 1 , whereas at both R.T.D. and a 1,000 R.T.D. it is 1 to 7 . It is further justified by the fact that $\beta$-lysin production is a common characteristic of the non-fibrinolytic phage-susceptible group (114 of 131 strains, $87.0 \%$ ), whereas only 14 of 74 $(18.9 \%)$ of the non-typable organisms showed this activity. It is also significant to note that $\beta$-lysin was not produced by any of the neomycin-sensitiver staphylococci, and it was an almost exclusive property of yellow, non-fibrinolytic, neomycin N resistant strains in this series.

It may be possible to use fibrinolytic activity as 20 marker characteristic in epidemiological studies of yellow non-typable staphylococci. In the presen study these organisms could be divided into two groups on the basis of this enzymatic activity approximately equal numbers falling into each? group. Thus, the use of appropriate cultural tests is not only of value in subdividing strains of staphy $\frac{\text { O }}{\mathbb{D}}$ lococci that are phage-susceptible, but provides the only routine means of effecting a subdivision of strainß that are not typable with the standard set of phages? 
None of the 552 strains studied was resistant to methicillin, cloxacillin, or fusidic acid, but 166 strains showed resistance to one or more of the other antibiotics, chloramphenicol, erythromycin, and novobiocin. Table IV relates these properties to neomycin resistance and pigmentation. Neomycin resistance is clearly not correlated to inhibition by any of the other antibiotics tested. From the results obtained it would seem that the pigmentation of a strain in no way influences its ability to manifest resistance to the range of antibiotics in common use, with the notable exceptions of neomycin and kanamycin. Thus, a third of both the yellow neomycinresistant and the orange and buff neomycin-sensitive organisms showed additional resistance. The antibiotic other than neomycin to which strains most commonly showed additional resistance was chloramphenicol, and even here only $21 \%$ of yellow neomycin-resistant and $15.7 \%$ of orange and buff neomycin-sensitive strains showed it. This is in marked contrast to the pattern of neomycin resistance within the yellow group, in which $78.0 \%$ were resistant.

\section{TABLE IV}

RESISTANCE OF 552 TETRACYCLINE-RESISTANT STRAINS OF STAPHYLOCOCCUS AUREUS TO NEOMYCIN, CHLORAMPHENICOL, ERYTHROMYCIN, AND NOVOBIOCIN

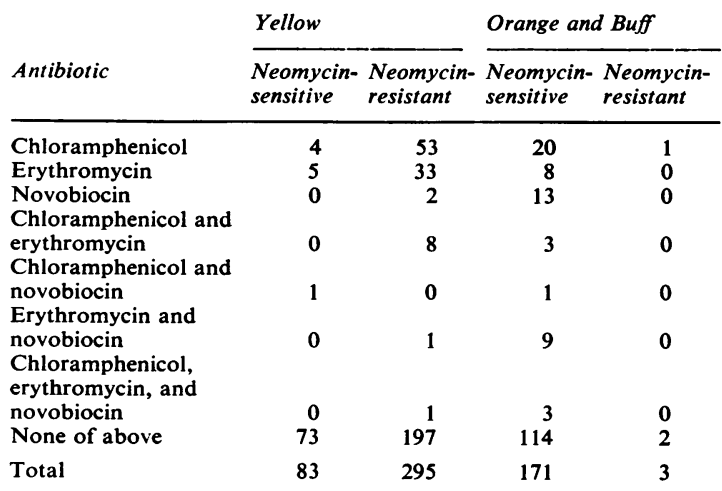

All strains resistant to penicillin, streptomycin and tetracycline; no strain resistant to methicillin, cloxacillin, or fusidic acid

These considerations lead us to suggest that mere exposure of staphylococci to neomycin or kanamycin has not played a significant causative part in the emergence of strains that are resistant to these antibiotics.

\section{DISCUSSION}

Neomycin-resistance amongst staphylococci is a characteristic almost exclusively confined to those strains that produce yellow pigmented colonies on glycerol monoacetate agar. Limitation of this property to yellow strains reinforces the view that yellow pigmentation is a valid marker characteristic for some epidemic strains of Staph. aureus (Turner and Willis, 1963; Willis and Turner, 1962), and provides further justification for the physiological subdivision of pathogenic staphylococci suggested by Willis et al. (1963, 1964). These yellow staphylococci comprise a phage-group-III-linked spectrum of related organisms that are characterized by their tetracycline resistance, their relative phage insusceptibility, and by their failure to produce a proteolytic enzyme recognized by its action on washed chocolate agar. In addition, most of the group are non-fibrinolytic, and these strains form $\beta$-lysin much more commonly than other staphylococci. The yellow group of organisms has a notorious epidemic potential in the hospital environment, and it is apparent that resistance to neomycin and kanamycin are additional properties of value in the delineation of this group. The phage reactions of these organisms have caused considerable confusion, and have tended to fragment what appears to us to be a homogenous group. We feel that a more useful and realistic subdivision of this natural group may be effected by the application of physiological studies, since these can emphasize both relationships and differences between organisms under examination.

The degree of susceptibility of yellow staphylococci to phage lysis is broadly related to neomycin resistance. This is brought out in Table $\mathrm{I}$, which shows that although non-typable yellow strains are almost always neomycin-resistant (Robertson, 1963), this property is shared, though to a lesser degree, by strains that are lysed at R.T.D. and at 1,000 R.T.D.

It is interesting to speculate about the cause of the neomycin-resistance exhibited by some strains of Staph. aureus. It may be that the emergence of neomycin-resistant staphylococci is linked to exposure to this or related antibiotics in hospital practice as suggested by Cohen et al. (1962). Consideration of the data presented here makes this unlikely. Thus, the distribution of neomycin resistance encountered within our wide range of tetracycline-resistant strains of staphylococci is both narrow and unusual; neomycin resistance occurred in about $80 \%$ of yellow staphylococci and in less than $2 \%$ of other strains. In contrast to this unique distribution of neomycin resistance, it will be recalled that tetracycline-resistant staphylococci of all colours show an equal predisposition to the development of resistance to chloramphenicol, erythromycin, and novobiocin. It might be argued that the yellow organisms are remarkable in their ability to acquire resistance to neomycin and related compounds, and may in 
consequence be selected out in the hospital environment, where neomycin is widely used. If this were indeed the case, then one might expect to encounter strains of staphylococci that were resistant to neomycin but sensitive to tetracycline. Moreover, neomycin finds its widest application in hospital practice, not in the treatment of staphylococcal infections but in the prophylactic preparation of the bowel in elective surgical cases. Patients of this type typically carry an 'out-patient' bacterial flora, and in our experience it is exceedingly rare to find tetracycline-resistant staphylococci, let alone the yellow variety, in persons on first admission to hospital. If indeed exposure to neomycin was a direct cause, acting either by selection or mutation, of the emergence of resistant strains of staphylococci, then one would reasonably expect to isolate orange and yellow tetracycline-sensitive, but neomycinresistant strains from these prophylactically treated patients. We have never encountered such a staphylococcus.

The association of tetracycline resistance with neomycin resistance is more apparent than real, since there is no evidence to suggest that cross resistance develops between these antibiotics (Kunin, Wilcox, Najarian, and Finland, 1958). These considerations suggest that there is a group of staphylococci that is inherently resistant to neomycin, or that agenciese? other than exposure to neomycin have been responsi-o ble for the emergence of strains resistant to itso action, agencies to which yellow strains must be particularly susceptible.

We wish to thank Dr. G. B. Ludlam for phage typingen the staphylococci, and we are indebted to Dr. W. Goldie and our departmental colleagues who made strains: available to us. Beecham Research Laboratories and $\vec{\omega}$ Leo Laboratories kindly supplied us with Orbenin ando Fucidin antibiotic discs, and Lederle Ltd. providedo generous financial support for the investigation.

\section{REFERENCES}

Cohen, L. S., Fekety, F. R., and Cluff, L. E. (1962). New Engl. J. Med., $\vec{\sim}$ 266, 367.

Goodburn, G. M. (1963). Personal communication.
Jacobs, S. I., and Willis, A. T. (1963). Lancet, 2, 459.

- and Goodburn, G.M. (1964). J. Path. Bact., 87, 151. Iudam, G. B., and Goodburn, G. M. (1963). 972.

Kunin, C. M., Wilcox, C., Najarian, A., and Finland, M. (1958). Proc. Soc. exp. Biol. (N.Y.), 99, 312.

Robertson, J. J. (1963). Lancet, 2, 333.

Temple, N. E. I., and Blackburn, E. A. (1963). Ibid., 1, 581.

Turner, G. C. (1963). Ibid., 1, 1156.

, and Willis, A. T. (1963). J. Path. Bact., 85, 395.

Willis, A. T., and Turner, G. C. (1962). Ibid., 84, 3.

- Jacobs, S. I., and Goodburn, G. M. (1963). Lancet, 2, 67. (1964). J. Path. Bact., 87, 157. 